\title{
Neurocybernetics, Codes and Computation
}

\author{
Roberto Moreno Díaz \\ Centro Internacional de Investigación en Ciencias de la Computación \\ Universidad de Las Palmas de Gran Canaria \\ Canary Islands, Spain \\ e-mail: rmoreno@ciicc.ulpgc.es
}

\begin{abstract}
Some fifty years back, a synergetic process took place which was to produce ever greater emphasis on the multi-disciplinary approach to science, relating to communication, coding, control and computation in human beings and machines. We attempt here to give a brief overview of the past evolution and future trends for some of the original concepts, mostly those pertaining to sensory coding, logical nets and neural computation to varying degrees of complexity. It is an attempt to make us all relive the excitement and the extraordinary force of the subjects together with the realms of inspiration still to be discovered in the work of the pioneers. It is also a modest contribution towards the commemoration of the Centenary of McCulloch's birth and the fiftieth anniversary of Wiener's book Cybernetics (1948)
\end{abstract}

\section{The Classics}

A doubtless partial perspective but, nevertheless, redolent in its conception, is that the nervous system is a network of partially interconnected systems which exchange messages, often in the form of commands. Each system, in itself, made up of a series of networks of computing units, rich in structure and functions, known as neurones. One of the greatest complications involved in understanding this network, even if only at the level of processing, coding and transmitting signals, is in unravelling the nature of the messages and, above all, their neural code. All of this and more, for there is also a need to come to a detailed understanding of the structures and the underlying wiring and links. In this quick overview of all sciences which come together to form Neurocybernetics, we shall look at what we already know and what we still have to learn about this fascinating subject, fascinating because its interdisciplinarity.

As we all know, Neurocybernetics took off in the Forties although many of the basic ideas had been being managed in philosophic and scientific circles since the times of the Ancient Greeks. From 1943 to 1945, a kind of synergetic process was started up, triggered as the result of three basic pieces of work: Norbert Wiener, 
Arthur Rosemblueth and Julian Bigelow's study (1943) on the nature of teleological processes where the crucial idea was that what was relevant in a homeostatic process was the information return and not the energy return via the feedback links.

Following this, came the work of the young British philosopher, Kenneth Craick, published in the form of a small book called On the Nature of Explanation in 1943, offered a pursuit of a Theory of Knowledge which would be contrastable like any other Natural science. He was not completely successful in achieving this aim but he did, however, establish the rational bases upon which all the theories and models of systems of artificial behaviour have since been built. Craick offered a clear and powerful frame work within which to express the acquisition, processing, storage, communication and use of knowledge.

And last but not least, the work of Warren McCulloch and Walter Pitts, $A$ Logical Calculus of the Ideas Immanent in Nervous Activity, which was published in 1943. They elaborated the concept of a "formal neuron" the response of which is, in fact, equivalent to a symbolic proposal with respect to the corresponding stimulus and which allows for a neural network to be considered as a logical system capable of handling symbols and elevating them to the level of the logic required for proposals. They came to the final conclusion that a network of formal neurons, with an effective infinite memory tape, can compute any number which is computable by a Turing Machine.

As of these studies, Neurocybernetics evolved with powerful input from the Theory of Communication of Shannon and key figures in the field of Computer Science such as Von Neuman, in the case of the latter, with application to questions of computability, performability, capacity for reproduction and reliability of functioning. McCulloch and Von Neuman were personal friends. McCulloch delighted a great deal in recounting the anecdote of how they began their work together on reliability of functioning, probabilistic logic and probabilistic computing.

McCulloch then held (in the Fifties) the chair of Psychiatry at the University of Chicago and one night, he and Von Neuman and some colleagues went overboard with the whisky. McCulloch suddenly stopped the conversation dead and commented something to the effect of "The thresholds of neurones are now at an all time low. Nevertheless, they are still computing reasonably reliably. What can there be in the brain, in its modular structure and links which makes it such a reliable piece of machinery in spite of failure in threshold levels and components?"

A magnificent piece of work called Agathe Tyche: The lucky reckoners offers us a fair overview of much of his philosophy with respect to ways of building reliable machinery from unsafe components. The classic by Cowan called Reliable Computation in the Presence of Noise and almost all of his later work on reliable computing was the result of the original concept of Von Neuman's as broadened by McCulloch. Reliable transmission had different roots although nowadays, and for 
some time now, these have become intertwined for the same simple reason as transmission and computing.

\section{Neurocomputing and Code}

One of the great aims of Neuroscience form the Fifties through to the end of the Sixties was to build up a type of Neuro-Physiological Epistemology, ie. a Theory of Knowledge with consolidated neuro-physiological bases. However, there were two major problems. On the one hand, the formal tools and the mathematics necessary to move from the experimental neurophysiological data to a theory of communication, processing and decision making in the brain, were as yet inexistent. On the other hand, most of the experimentalists did not plan their experiments using the correct theoretical constructs. Therefore, the results were often short reaching and scarcely useful in that they did not allow for building even a minor theory at the primary level corresponding to sensorial perception.

Jerome Lettvin, the outstanding example of a Doctor in Psychiatry who later was to become Professor of Communications in the Department of Electrical Engineering at the MIT, was a student of McCulloch's in Chicago and was completely absorbed by his ideas. At the end of the Fifties, and with the help of the anatomist, Humberto Maturana. who was originally from Chile and who had been trained in the school of Ramón y Cajal and Lorente de No, Lettvin picked up the concepts relating to Neuro-physiological Epistemology and tried to crack the process of codification and transmission of data in the visual system of an animal of scarce brain but highly successful in survival in the environment, the frog. Lettvin, Maturana, McCulloch and Pitts produced an article in 1959 which is a masterpiece of Neuro-science, of extraordinary quality at the anatomical, neuro-physiological or rather Electro-physiological levels, to be more precise, with data obtained with firstgrade electronic instruments, much of which had been designed and built by themselves. I am, of course, referring to the piece of work What the frog's eye tells the frog's brain? which was first published in the Proceedings of the IRE, present IEEE since there was no professional physiological journal which was willing to accept it originally.

At this juncture, it would be appropriate to talk about the neural code. Talk about signals, noise, and more importantly, codes with respect to the physiology of the brain first appeared when the procedures for registering the Electro-physiology were perfected in two different directions: first, in the design of micro-pipettes which were adequate to the task of registering singular fibers, axons, rather than isolated fibers and when electronic technology, as the result of World War II allowed for the design and constructions of amplifiers with a high level of sensitivity and a low level of noise. The first experiments designed at cracking the neural code were carried out by "tapping" the optic nerve of lower vertebrates (amphibians) until sure of registering one sole fiber and then searching in the retina, with a small light spot, where, when and how a response was obtained in the form of a series of 
pulses. The "where" was given the name of the receptive field of the fiber which was broadened later to include the points at which, when a further stimulus was given, the response to the first stimulus diminished. Nowadays, fortunately, we can sum this all up in a few words thanks to the terminology and the formalisms contributed by the signal theory.

The brain is a layered anatomical structure which, to the effects of this paper, we can consider to resemble a computer or a signal processor organised by levels and layers of computational units, the neurons, which communicate laterally at each and every level and send their orders-messages to the following layer whilst receiving a certain level of feedback from the same. In the cerebral cortex, the number of connections and the possible codes make it difficult, if not impossible, to control the stimulus, the input signal which arrives at the system except perhaps in certain areas of the primary sensorial cortex. Besides, the retina in vertebrates represented an "evolutionary gift" for the researchers of the later part of this century. The retina has the same anatomical-functional structure by layers as the brain. Of all the sensors, which a superior organism projects towards the outside world, the retina is the only one which retains the basic structure of the cortex: the eyes are like two small pieces of cerebral cortex which look out onto the world. It is as if, bearing in mind the obvious exaggeration, two parts of a central computer bank were separated and converted into peripheral bases. We have to be careful of making analogies here for very many reasons: the first is that our present computer systems are not layered and as far as we can see form the state of the art, they are far from ever being so. The other reasons follow from this first reason and basically have to do with the deep break, key as it has so turned out, between computing codes in artificial systems and the codes of neurons in Nature.

In the retina, there are three distinguishable layers of computing which run from the photo-receptors to the bi-polar cells and form these, fanning out over the field of reception, to the gangliar cells whose axons meet to form the optic nerves, which in higher vertebrates, then branch out after the optic chiasma towards the geniculated lateral bodies, ending up in the visual cortex, area 17, in the occiput after having received additional information from other brain centres. In the frog, the whole system is much simpler since the gangliar axons project towards the optic tectum or roof which seems to take decisions, insofar as the reticular formation allows for the same, by way of command and control systems common to all vertebrates. But let us leave the frog aside, which has a highly non-linear visual system. Research into the retina of higher vertebrates has been carried out basically with birds (the dove), cats and monkeys. With a range of fairly perceptible retinal illumination, most of the gangliar cells of the cat, less so in the dove, and all in the monkey behaved linearly with linearity measured as the relationship between the visual stimulus-sporadic- in the field of reception of a gangliar cell, and the response in terms of pulse frequency or, in some cases, the lapse between pulses on the axon which forms part of the optic nerve. 
This situation, nowadays, is what all-young mathematicians, physicists, engineers and computer scientists dream that their boss will order them to research. But, in fact, the situation, seems to be not so simple because, first, you have to read everything which has been published by experimentalists who talk about on receptive fields (the cell "fires" when the luminous spot is lit in some part of the receptive field) OFF cells, when the cell backfires and the spot is turned off, centre ON cells and peripheral OFF cells and viceversa, cells with ON-OFF rings, cells which have an additional ring in the receptive field which is sometimes an enhance (it increases the response) and others an inhibitor (it decreases the response). All of this and some non-linearity thrown in for good measure, although not excessively impertinent in nature (with "diode" type actions, much of this is aproximately balanced).

However, fortunately, thanks to modern concepts of spatio-temporal processing it is easier, as I already indicated, to express what most gangliar retinal cells do according to Electro-physiology. They capture data in a space and time constructed by the photo-receptors and the bi-polar and other lateral cells in closed concentric volumes, give them positive or negative weightings according to the area, add and encode the results in a series of pulses which are modulated in frequency (see fig. 1, for illustration of the center-periphery concept, and fig. 2 for recent dendro-dendritic computation). This is the sum total of fifty years of conventional retinal Electro-physiology, except for some slight non-linearities in specialised gangliar cells. And, of course, except for the conceptual content cited in the work of Lettvin et al., in the IRE in 1959.

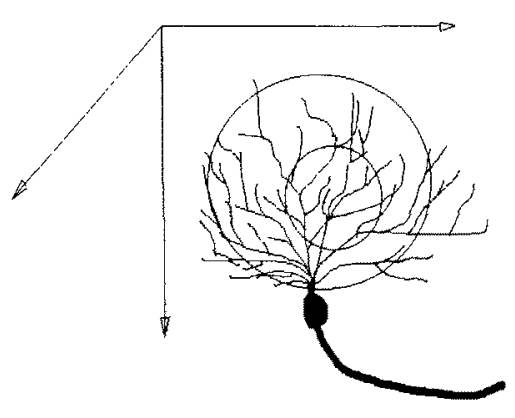

IDEALIZED SPACE-TIME INPUT (INNER PLEXIFORM LAYER)

CENTER-PERIPHERY ZONES FOR DATA ACQUISITION

GANGLION CELL (COMPUTATION AND CODING)

AXON (OPTIC NERVE)

Fig.1. llustration of Center Periphery receptive field volumes for a Gangliar Cell 
Let us go back for a second, if we may, to the publication of the Institute of Radio Electronics in 1959. What was crucial in the experiments in this work by Lettvin et al., was that they decided to leave the frog as close to its natural environment as possible and to stimulate it with signals as close as possible again to what a frog would receive as signals on an everyday basis. Then, in the purest tradition of Electro-physiology, they registered near to the tectum opticum. It is of course and elementary fact that a frog, in a pool, has never received throughout its evolution small dots of light which flash on and off. It is highly illustrating to reread how they classified the gangliar cells in the retina of the frog: cells which responded only to quick decreases in light entering peripheral zones: cells which responded to any change but selectively to more local changes; and, above all, the famous Group II of gangliar cells which only responded to small dark objects which moved centripetally over the field of reception, the cells which detected "bugs" or possible food.
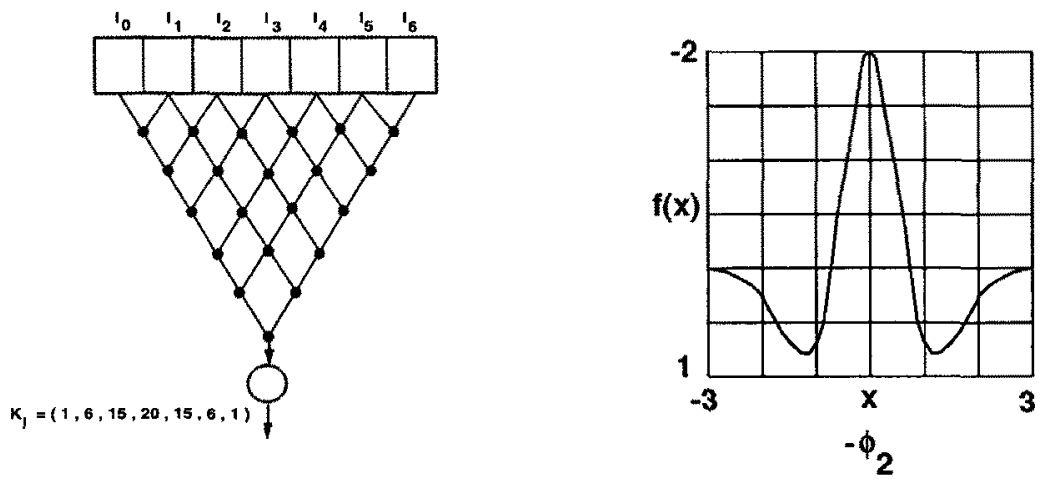

Fig.2. Examples of linear dendro-dendritic computation which generates a Hermite gaussian and a " mexican hat" receptive field.

If we stick at the level of pure response to stimulus and we apply the relatively powerful machinery of signal analysis and processing, we can come to a pre-theory with respect to the retina of the frog and what is says to the brain. This was done in 1966 and has been rectified several times since until we have come to the conclusion that, even at the level of the retina, the tool is not as powerful as it seems, above all if we are dealing with vertebrates which are not low down on the phylogenetic scale and, as always, with reference to the retina. It is simple: over evolution, a magnificent advantage was found, in vertebrates, of projecting the incipient brain to the eyes, specialising the cells of the latter to detect what was vital (lower vertebrates) then, when the brain grows, withdrawing the interpretive function and decion making from these organs in such a way that, in the monkey, and possibly in human, all major function is positioned in the cortex, well beyond the visual cortex, leaving the retina as a good sample of the structure of the brain but without the great powers that it exerted before. Which, in a certain way, is of great advantage, 
allowing for many people to study optic properties such as trnsduction, inhibition and lateral interaction, its effectively as a transductor, by using conventional tools differential- integral equations with controlled non-linearities allowing us to arrive at results and even theories which seem to be satisfactory.

However, going up the evolutionary scale, the problem of Epistemology retreated to the cortex. Or, let me put it another way, as formulated by Lettvin and colleagues but slightly modified -what does the eye of the monkey say to the brain? Looking at the responses of the gangliar cells of the retina in the monkey, it would seem that the eye of the monkey does not say anything tremendously complicated. In countless situations, it seems as if the cerebrum dictates what the eye says. Which, when we come down to it, is fine because we are going to give various generations work to do trying to find out what the eye says to the brain and what the basic granules, the neurons, have to say.

\section{Neural Networks}

Let us now consider the theory of neural networks, which has risen from the ashes with renewed energy, as of some ten years back, with the importance of everything relating to distributed computing and connectivism.

Around 1965, some thirty years back, the office of McCulloch in the Electronic Research lab at the MIT was a kind of breathtaking classroom both for the quality of the science which was produced as for the incredible people who filed through it. All of the greats of Cybernetics visited the same: Colin Cherry, Donald McKay, Patrick Meredith, Von Foester, Gordon Pask, Eduardo Canianello, to name only a few. The well-known names of Marvin Minsky and Semour Papert set up the MAC project in Artificial Intelligence in a nearby lab. It was a young mathematician, Manuel Blum who had discovered the theory of neural networks with the interaction of afferents, together with another young mathematician, Michael Arbib.

After the problems with respect to the reliability of functioning, which reached some acceptable solutions, the theory of neural networks faced up to the question of dynamic memory. The problem refers to oscillations in the networks, expressly constructed to provoke controlled oscillations, which serve as a support to the dynamic storage of information. The initial logical problem was to find the maximum theoretical number of ways of oscillation in a non-linear, discrete an arbitrary network of $\mathrm{N}$ formal neurons. Schnabel calculated it and it is a number which grows extraordinarely when the number of formal neurons is increased. For example, for two neurons, there are twenty oscillation modes: for three, there 6.024 ie, three neurons could "store" 6.924 different models, each of which could be evoked by different external bays or input. We say, "it could" because we still have to show that a network of fixed anatomy could be designed that incorporates 
all the modes of fluctuation. This was demonstrated in 1966 via the theorem of synthesis and using formal neurons with afferent interaction introduced by Blum.

By the year 1969, the theory of formal Neural Networks was considered, from the logical perspective, to be a closed matter above all after the introduction of the so-called functional matrices which allowed, transparently, the demonstration of equivalence between determinist and probabilistic robots and networks of formal neurons with feedback, via constructive theorems. There was only one formal gap, discovered in 1977 by a student at the University of Zaragoza and it consisted in the fact that certain probabilistic machines had no counterpart in the logical networks of formal neurons unless an additional codifier was incorporated into the network, of a probabilistic type, previous to the input to the networks ie. unless the "outside" world (outside the neural) had a non-deterministic nature and, what is worse, a nature which depended on the structure of the network of formal neurons. In other words, that there are probabilistic robots which cannot be duplicated in the networks of formal neurons with afferent interaction. Although, in fact and in the practical totality of the applications, the subject is not completely without its relevance, from the theoretical perspective, it is inadmissible since we could not defend that the logical model of McCulloch and Pitts were an appropriate model to represent the activity of the brain at the computational level of coding and communication.

This gap was acknowledged, but the subject was parked due to the fact that neural networks suffered a fall in scientific interest from the end of the Seventies through to the mid Eighties. In 1983, a doctorate student in Maths, took up the subject again and proved that if interaction between axons was admitted - the output channels of the neurons - in an intercommunication process which may take place through the medium, in a network of hierarchizised formal neurons, then the theory was complete ie. a network of formal neurons with feedback would duplicate any arbitrary robot, be it deterministic, probabilistic or non-deterministic. This effect of output interaction was added elegantly to the interaction of afferents - input - of Blum dating back to 1962. This finishes the so called McCulloch's Program I, the logical program (see fig.3).

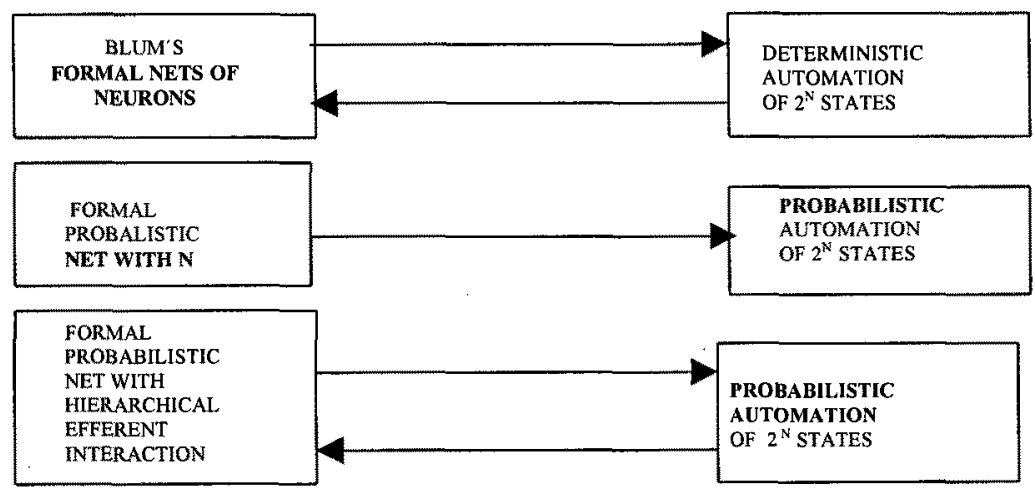

Fig.3. Illustration of the theorems of equivalence between formal neurons and automata. (Program I) 
McCulloch's Program II is more realistic and can be considered as brain theory at the level of Systems Sciences. The prototype paper is the 1947 paper by he and Pitts entitled "How we know Universals", as well as his and Kilmer's subsequent work on modelling the reticular formation. Actually, as Norbert Wiener says in the Introduction to his book Cybernetics, McCulloch was after an apparatus to enable to read the printed page by ear, which, because the necessary invariances, was a definite analogue of the problem of perception of form, of Gestalt. $\mathrm{He}$ designed a device that made von Bonin ask if it was a diagram of the fourth layer of the visual cortex. A typical neurocybernetic solution.

Program II can be simply stated as follows: from a sybsystem of the nervous system, define the most precisely its functions and try to find a cooperative, reliable granular structure to perform said functions. That will be a true theoretical neural net. Program II can be formulated for the artificial as well, so that it provides for systematic reasonable ways to solve problems by means of artificial "neural nets" of computing modules.

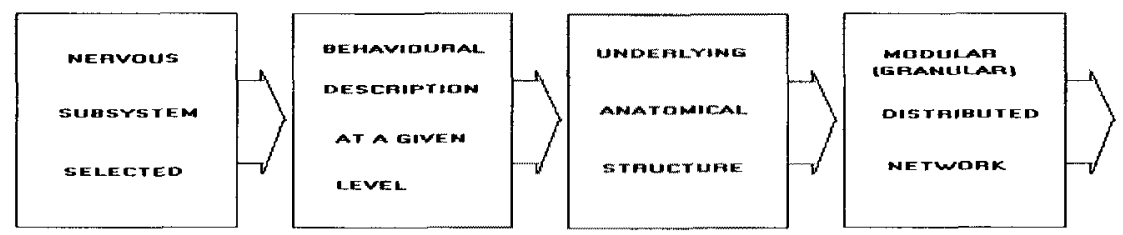

Fig. 4. Proceeding according McCulloch's Program II

We indicated that in the Eighties, there was an upsurge in neural computing which, I believe, was due to one basic cause, the growing availability of microcomputers at a very low cost so that hundreds and even thousands of them could be linked up in parallel processing networks, each with functions much more complex than the formal neurons of McCulloch and Pitts and the addenda. Anyway, we should not lose sight of the fact that the classic theory is complete at a logical level and by offering greater computing potential to the basic units, the maximum we arrive at is a reduction in the number of units needed for the practical working of an artificial system apart from an increase in the speed of design. The only crucial element which had not been contemplated and which was easy to incorporate - and was incorporated in the famous Perceprons of the $60 \mathrm{~s}$ - was the capacity of modification of synaptic weighting through learning.

As it is well known, this resurgence of neural networks as systems of distributed granular computing is finding application in technological fields ranging 
from processing and treatment of signals (voice, image), systems of artificial vision, in robots and in controls. For further illustration, see the proceedings of the last International Workshop on artificial neural nets (see Mira and Sandoval eds.1995, Mira et al eds 1997). We howeverbelieve that most works on artificial neural nets are irrelevant ways of solving problems using non-optimal tools. It is our believe that significant progress in artificial neural net theory (or modular distributed computation) requires to proceed strictly as in Program II.

\section{The Levels of Description}

The classic theory continues to allow, nevertheless, for successive refinements pertaining to two basic questions as our knowledge of the nervous system advances: first, what are the counterparts or models, bearing in mind the more modern tools of signal processing, which can allow us greater information with respect to the behaviour of non-peripheral zones of the nervous system, such as the auditive and the visual cortex and second, how can we "refine" the theorems of synthesis, in order to adapt them, not logically but rather structurally to the reality, ever less of a mystery, of the anatomy of the brain.

Groups all around the world and in this country have sprung up to this effect, working seriously on neural nets, artificial neurons, cooperativity, visual data processing and learning and acquisition of knowledge. This activity has recently been fired thanks to the aforementioned Renaissance of the 80s although, unfortunately, with not much more knowledge than before which has often led to rediscover the Mediterranean (see Moreno-Díaz 1995).

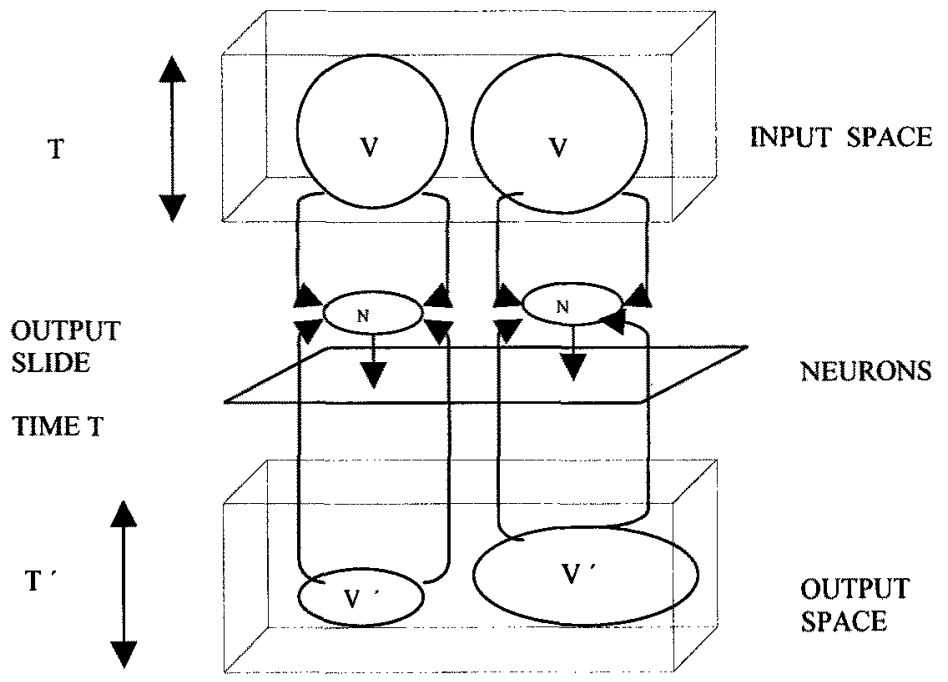

Fig.5. Illustration of generalized neurons, which compute on data from symbolic input and output spaces 
What are the limits of the classic theory of neural nets? The classic theory moves on the wavelength of logical language and allows for valid conclusions to be reached although with adequate extensions it could cope with phenomena of slightly lover or higher range. It is not the total description of the behaviour of the brain but it is one of many necessary descriptions. The neural function is a really complex phenomenon and its characterisation requires, as a norm, meticulous approaches both at the level of tools and methods to be applied as in accepting or choosing the parameters which are considered necessary when describing and trying to explain this function. Also care should be taken when considering the scope of possible validity of conclusions reached via the theoretical and experimental approaches adopted. This is equivalent to saying that any theory with respect to the nervous system is limited $a$ priori by the conceptual tools. To exaggerate, we cannot attempt to explain the capacity for resolution of problems of the nervous system using, for example, non-linear differential-integral equations. Nor can we delve deeper into the properties of the neural membrane using the logic of relationships.

If we remember the registering of impulses of potential action, which we spoke about earlier when talking about the retina, we cannot deny the historic role-played by action potential since the Fifties in that they have allowed for a physical knowledge of the carrier substratum of messages. But it is illegitimate to work from them to deduce high level properties or to try to build functional models of the brain. It would be, albeit an unfair comparison like using statistics of the pulses which appear in a data bus or computer commands to deduce the algorithmic base of the programme solving a problem in RAM.

We can sum up this structure of Neurocybernetic levels in a way which indicates which are the appropriate tools for each level bearing in mind that a notable change in level cannot be allowed in the theory without changing tools. But, if prudent, in the practical research into the brain and artificial machines which we wish to make work like the brain, we can skip the level slightly.

The most basic level (where computational machines still do not appear, strictly, apart from as tools) is the level of the neurotransmitters, the membrane phenomena and action potential. The tools are Biochemistry and Biophysics. Then comes Biophysics of Neural codes and multiple codes - this is a word used in neurophysiology to indicate multiplex - then we move onto Biophysics and Signal Processing. We continue through sensorial codes, decodification in effectors motor and glandular action - and the code of advanced peripheral neurons such as the previously mentioned gangliar cells in the retina. We are now in the realm of Signal Theory almost at the level of logic. Then, we have the neural net level, the interaction of input and output of the neurons themselves, and the coordination of the output -effectors. We are now at the level of the Language of Logic bordering on Symbolic Languages and, finally, we come to the central cortex neural code, the cooperative processes between masses of brain tissue, the extraction of Universals 
and the social processes of interaction between neuron masses. We are at the level of Symbolic language.

I shall rewind Biophysics, Biochemistry, Processes and Theory of Signals, Logical Language and Symbolic Language. This could be said to be the title to the headings for the visual process From the Retina to the Associative areas in the Brain.

This representation by levels, which, in practice, are not separate which interact in a quasi continuum reveals in itself the multi-disciplinary nature of neurocybernetics, which anyone who wants to understand a little with respect to the brain, or to design and build machines which function like brains, must study.

It appears more and more evident that there is a basic difference, both in structures and codes, between natural and artificial machines. There are many who insist upon labouring the point and one of the most sensible articles which I have read and discussed personally is what J. Mira Mira and colleagues, now in the Open University in Madrid, have done and do with respect to the adequate representation of the brain's high level functions. The computational structure by layers is an evolutionary property enjoyed by the nervous system. In this structure, the physical shape, even from the point of view of the theory of the signal, the code between the layers, appears to start from the sensors and peripheral effectors to the more central zones of the cortex. In the brain, electric stimulation in high zones of the process of computation (the cortex) triggers, despite the simple nature of the stimulus, complex and frankly coherent perceptions and behaviour. This does not occur with conventional computers. We cannot, within the analogy cited before in another context, stimulate the bus of the computer with a simple sequence of pulses and hope that instead of the coherent list of businesses with which we have contacts, to receive coherently the monthly payroll just be because both are in the RAM. The development of artificial machines which, somehow, incorporate these and other properties of the ever more amazing nerve tissue, is the uniting drive for all of us who work, at one level or another, within neural computing, granular computing or layered connectivism.

\section{Selected References}

Lettvin, J.Y.; Maturana, H.R.; McCulloch, W.S.; Pitts, W.H. (1959): What the frog's eye tells the frog's brain. Proc IRE 27, pp 400-415.

McCulloch, W.S. and Pitts, W.H. (1943): "A logical Calculus of the Ideas Immanent in Nervous Activity". Bull. Math. Biophysics, 5, pp 115-133. 
McCulloch, W.S. (1959): “Agatha Tyche: of Nervous Nets The Lucky Reckoners". Mechanization of Thounght Processes: Proceedings of a Symposium Held at the National Physical Laboratory, November, 24-27, 1958, $\mathrm{n}^{\circ} 10$ Vol II, London, Her Majesty's Stationery Office, pp 611-634.

McCulloch, W.S. and Moreno-Díaz, R. (1967): "On a Calculus for Triadas", in "Neural Networks", Caianiello, ed. Springer-Verlag Berlín, Heidelberg. New York, pp 78-86.

McCulloch, W.S. (1969): "Regenerative Loop". The Journal of Nervous and Mental Disease, 149, pp 54-58.

Mira Mira, J. (1971): "Modelos Cibernéticos de Aprendizaje". Tesis Doctoral. Facultad de Ciencias de la Universidad de Madrid.

Mira Mira, J.; Delgado, A. (1983): "On cooperative languages for survival and nerual decision making". Proc. Int. Symp. on Man in High Tech Env. Namur. pp 569-581.

Mira Mira, J.; Moreno-Díaz, R. (1984): “Un marco teórico para interpretar la función neuronal a altos niveles". In Biocibernética. Moreno-Díaz, R.; Mira Mira, J. eds. Siglo XXI. Madrid, pp 149-171.

Mira, J.; Sandoval eds., (1995): "From Natural to Artificial Neural Computation". Lecture Notes in Computer Science, vol 930. Springer-Verlag, Berlín Heidelberg.

Mira, J.; Moreno-Díaz, R.; Cabestany, J. eds. (1997): "Biological and Artificial Computation: From Neuroscience to Technology. Springer Verlag. Berlin. Lecture Notes in Computer Science no. 1240.

Moreno-Díaz, R. and McCulloch, W.S. (1968): "Circularities in Nets and the Concept of Functional Matrices" in L. Proctor, ed. "Biocybernetics of the C.N.S." pp 145-150. Little and Brown. Massachusetts.

Moreno-Díaz, R. (1971): "Deterministic and Probabilistic Neural Nets with Loops". Mathematical Biosciences 11, pp 129-136.

Moreno-Díaz, R.; Hernández Guarch, F. (1983): "On the biological formal counterparts of logical machines". Kybernetes, 12, pp 183-185.

Moreno-Díaz, R., (1995): "De los orígenes y evolución de la teoría de redes neuronales" en Computación Neuronal: S. Barro; J. Mira, eds. Cap. V. Servicio de Publicaciones e Intercambio Científico. Universidad de Santiago de Compostela.

Moreno-Díaz, R.; Mira-Mira, eds. (1995): Brain Processes, Theories and Models. The MIT Press. Cambridge MASS. USA. 
Neumann, J. von (1956): "Probabilistic Logics and the Synthesis of Reliable organisms form Unreliable Components". in "Automata Studies", Shannon and McCarthy, eds. Princenton University Press, Princenton, New Jersey.

Rosemblueth, A.; Wiener, N.; Bigelow, J. (1943): "Behaviour, Purpose and Teleology" Phylosophy of Science. Vol 10.

Rubio Royo, F. (1969): "Modelos del proceso visual en la retina de los vertebrados. Simulación en ordenador". Tesis Doctoral. Universidad de Madrid.

Sutro, L. (1966): "Sensory Decision and Control Systems". Instrumentation Laboratory R-548. MIT, Cambridge, MA.

Wiener, N. (1948): Cybernetics. The Technology Press. John Wiley and Sons, Inc. New York. 